\title{
Introduction to AGG and EMF Tiger by modeling a Conference Scheduling System
}

\author{
Enrico Biermann • Claudia Ermel · Leen Lambers • \\ Ulrike Prange · Olga Runge - Gabriele Taentzer
}

Published online: 26 May 2010

(C) Springer-Verlag 2010

\begin{abstract}
In this paper, we focus on the Conference Scheduling System, a case study at the Tool Contest of Graph-Based Tools (GraBaTs) 2008. We took part in the contest with our graph transformation tool AGG and the ECLIPSE-based EMF model transformation tool EMF TIGER. We present the features of both tools and evaluate their abilities to model the Conference Scheduling System and to deal with additional contest assignments like model instance generation, property verification, and interoperability.
\end{abstract}

Keywords Graph transformation tool · Graph transformation case study · AGG · EMF transformation . Eclipse EMF

\section{Introduction}

Graphs are a general kind of models which have been used in various fields of computer science. On the one hand, they are well-suited to formally describe complex structures. On the other hand, the underlying structure of models, especially visual models, can be described best by graphs, due to their multidimensional extension. Graphs can be manipulated by

E. Biermann · C. Ermel $(\bowtie) \cdot$ U. Prange $\cdot$ O. Runge

Institut für Softwaretechnik und Theoretische Informatik,

Technische Universität Berlin, Berlin, Germany

e-mail: claudia.ermel@tu-berlin.de; emftiger@tfs.cs.tu-berlin.de

L. Lambers

Hasso-Plattner-Institut für Softwaresystemtechnik, GmbH,

Potsdam, Germany

e-mail: leen.lambers@hpi.uni-potsdam.de

G. Taentzer

Fachbereich Mathematik und Informatik, Philipps-Universität Marburg, Marburg, Germany

e-mail: taentzer@informatik.uni-marburg.de graph transformation in a rule-based manner. Considering current trends in software development such as model driven engineering and model-integrated computing, there is a need to describe model manipulations, model evolution, model semantics, etc. in a precise way. Recent research has shown that graph transformation is a promising formalism to specify model transformations.

Naturally, tools are crucial for the promotion of graph transformation in industry. Currently, a variety of tool environments exist for different graph transformation approaches, some of which are used in the area of model transformation [21]. In this paper, we present the potentials and limits of two tools by modelling the common application Conference Scheduling System, the live case study at the Tool Contest of Graph-Based Tools (GraBaTs) 2008 in Leicester [25]. ${ }^{1}$ All presented solutions (except the extension of AGG by an export to the DIMACS format) have been developed during the live contest which took one afternoon.

The tools in the focus of this paper are the well established graph transformation environment AGG [27,29], developed and extended over the last 15 years at TU Berlin, and the recently developed tool, EMF TIGER [4,31] (Transformation generation), a modeling and code generation framework for ECLIPSE applications based on EMF data models and graph transformation concepts.

The graph transformation research community has built up a significant body of knowledge over the past 30 years and, in addition to the theoretical base, several practical application lines have been established. The research area of model transformations has recently been identified as a key subject in model-driven development. The development goal

\footnotetext{
1 The authors received the GraBaTs 2008 Awards for the Best Live Contest Solutions in the categories Instance Generation and Interoperability, see http://fots.ua.ac.be/events/grabats2008/awards.html.
} 
of EMF TIGER is to foster interaction between the graph transformation and the model transformation community in order to facilitate exchange of results and challenging problems. Graph transformations could offer an elegant theory and powerful concepts for the model-driven engineering of software systems, while the software engineering community can generate interesting challenges for the graph transformation community. Therefore, there is a need for strong interaction and inter-operation between these communities: the interchange of ideas, problems, and solutions will lead to major advances in both fields.

Both of our tools come along with a visual interface to specify modeling languages (by type graphs in AGG, by EMF models in EMF TIGER) and transformation rules. AGG supports several kinds of validations which comprise graph parsing, consistency checking of graphs as well as conflict and dependency detection in transformations by critical pair analysis of graph rules.

The power of EMF TIGER lies in the generation of Java code which can be freely embedded in existing applications relying on EMF models. Another advantage is that EMF Tiger directly works on EMF models. Moreover, it is very easy to extend the generated Java code, e.g. to define rule control structures or (parts of the) matches from rules to model instance graphs. We demonstrate the features of EMF TIGER by modeling the instance generation assignment of the Conference Scheduling case study.

The paper is structured as follows: In Sect. 2, we review the basic notions of the formal framework of algebraic graph transformation. We introduce our tools AGG and EMF TIGER in Sect. 3, and present the solutions to different assignments of the Conference Scheduling case study in Sect. 4. Related work is compared to our tools and the underlying approaches in Sect. 5, and Sect. 6 evaluates the results of the case study, discussing the strong and weak points of both tools and giving directions for future developments.

\section{Basics of algebraic graph transformation}

In this section, we review the basics of the formal framework of algebraic graph transformation [13]. The main concepts of this framework which are relevant for both of our tools are typed graphs, graph grammars, transformations and constraints. System states of our conference scheduling case study are modelled by typed graphs.

Definition 1 (Typed Graphs) A graph $G=(N, E, s, t)$ consists of a set of nodes $N$, a set of edges $E$, and functions $s, t: E \rightarrow N$ assigning to each edge $e \in E$ the source $s(e) \in N$ and target $t(e) \in N$.
A graph morphism $f: G \rightarrow G^{\prime}$ is given by a pair of functions $f=\left(f_{N}: N \rightarrow N^{\prime}, f_{E}: E \rightarrow E^{\prime}\right)$ which is compatible with source and target functions.

A type graph $T G$ is a distinguished graph where nodes and edges are considered as node and edge types, respectively. A $T G$-typed, or short typed, graph $\bar{G}=(G, t)$ consists of a graph $G$ and a graph morphism $t: G \rightarrow T G$, called typing morphism of G. Morphisms $f: \bar{G} \rightarrow \overline{G^{\prime}}$ of typed graphs are graph morphisms $f: G \rightarrow G^{\prime}$ which are compatible with the typing morphisms of $G$ and $G^{\prime}$, i.e. $t^{\prime} \circ f=t$.

For simplicity, we abbreviate $\bar{G}=(G, t)$ by $G$ in the following. Moreover, the approach is also valid for attributed and typed attributed graphs where nodes and edges can have data type attributes of a given data type $D$ (see [13] for more details). Figure 2 in Sect. 4.1.1 shows the type graph $T G$ of our conference scheduling case study, whereas Fig. 7 in Sect. 4.1.3 shows the initial system state as $T G$-typed graph.

The dynamic behaviour of systems is modelled by rules and transformations of a typed graph grammar:

Definition 2 (Typed graph grammar) A typed graph grammar $G G=\left(T G, G_{\text {init }}, P\right)$ consists of a type graph $T G$, a $T G$-typed graph $G_{\text {init }}$, called initial graph, and a set $P$ of rules (also called productions). Each rule $r \in P$ is given by a span $(L \leftarrow I \rightarrow R)$, where $L, I$ and $R$ are $T G$-typed graphs, called left-hand side, right-hand side and interface, respectively. Moreover, $I \rightarrow L, I \rightarrow R$ are injective typed graph morphisms where in most cases $I$ can be considered as intersection of $L$ and $R$. A rule $r \in P$ is applied to a $T G$ typed graph $G$ by a match morphism $m: L \rightarrow G$ leading to a direct transformation $G \stackrel{r, m}{\Longrightarrow} H$ via $(r, m)$ in two steps: at first, we delete the match $m(L)$ without $m(I)$ from $G$ to obtain a context graph $D$, and secondly, we glue together $D$ with $R$ along $I$ leading to a $T G$-typed graph $H$. More formally, the direct transformation $G \stackrel{r, m}{\Longrightarrow} H$ is given by two pushout diagrams (1) and (2) in the category Graphs TG of $T G$-typed graphs, where diagram (1) (resp. (2)) corresponds to gluing $G$ of $L$ and $D$ along $I$ (resp. to gluing $H$ of $R$ and $D$ along $I$ ).

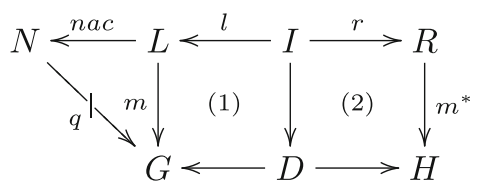

Note that pushout diagram (1) in step 1 only exists if the match $m$ satisfies a gluing condition w.r.t. rule $r$ which makes sure that the deletion in step 1 leads to a well-defined $T G$ typed graph $D$. Moreover, rules are allowed to have negative application conditions (NACs) given by a typed graph morphism nac : $L \rightarrow N$. In this case, rule $r$ can only be applied at match $m: L \rightarrow G$ if there is no injective morphism 
$q: N \rightarrow G$ with $q \circ n a c=m$. This means intuitively that $r$ cannot be applied to $G$ if graph $N$ occurs in $G$. A transformation $G_{0} \stackrel{*}{\Longrightarrow} G_{n}$ via $P$ in $G G$ consists of $n>0$ direct transformations $G_{0} \Rightarrow G_{1} \Rightarrow \cdots \Rightarrow G_{n}$ via rules $r \in P$.

An example showing one rule $r=(L \leftarrow I \rightarrow R)$ without NAC and one rule with a NAC nac $: L \rightarrow N$ is given in Fig. 3 (note that our rule figures do not show the interface $I$ which always consists of the nodes and edges which are present in both $L$ and $R$, as indicated by equal numbers. Concerning the representation of NACs, for simplicity we only show the additional part $N A C$ of $N$ in the left which extends $L$. All graph morphisms are inclusions.

In order to model consistency properties of system, we use graph constraints. An atomic T G-typed graph constraint is given by a $T G$-typed graph morphism $f: P \rightarrow C$ and consists of a premise $P$ (drawn as left-hand side of the constraint) and a conclusion $C$ (drawn as right-hand side). It models an if (precondition) then (conclusion) construct. Atomic constraints can be combined to more complex graph constraints by boolean operators and connectors (e.g. $\neg G \wedge H$ ). A graph constraint $G C$ is satisfied by a TG-typed graph $G$, written $G \models G C$, if for each injective graph morphism $p: P \rightarrow G$ there is an injective graph morphism $c: C \rightarrow G$ with $c \circ f=$ p. A sample atomic graph constraint can be seen in Fig. 9.

\section{Modeling tools}

\subsection{AGG}

AGG [27,29] is a well-established tool environment for algebraic graph transformation systems, developed and extended over the last 15 years. Graphs in AGG are defined by a type graph with node type inheritance and may be attributed by any kind of Java objects. Graph transformations can be equipped with arbitrary computations on these Java objects described by Java expressions. The AGG environment consists of a graphical user interface comprising several visual editors, an interpreter, and a set of validation tools. The interpreter allows the stepwise transformation of graphs as well as rule applications as long as possible. AGG supports several kinds of validations which comprise graph parsing, consistency checking of graphs, and conflict and dependency detection by critical pair analysis of graph rules. Furthermore, checking the applicability of rule sequences is supported, as well. Applications of AGG include graph and rule-based modeling of software, validation of system properties by assigning a graph transformation based semantics to some system model, graph transformation based evolution of software, and the definition of visual languages based on graph grammars.

\subsection{EMF TIGER}

EMF Tiger [4,31] (Transformation generation) is a recently developed Eclipse plug-in ${ }^{2}$ supporting modeling and code generation for EMF model transformations, based on structured data models and graph transformation concepts. Although EMF provides basic operations for modifying EMF based models, it is still difficult to define more complex operations on these models. The goal of EMF Tiger is to provide the means to graphically define rule-based transformations on EMF models. Rule applications change an EMF model instance in-place, i.e. an EMF instance model is modified directly, without copying it before. Moreover, control of rule applications is supported by EMF Tiger, as well as pre-definition of (parts of) the match. EMF Tiger currently consists of a graphical editor for visually defining EMF model transformation rules, and a compiler, generating Java code from these transformation rules to be included into existing projects performing EMF model transformation. It also contains an interpreter which translates EMF transformation rules to AGG. This interpreter is useful for verification purposes. Since EMF TIGER is based on graph transformation concepts it can be verified along important transformation properties.

\subsection{Differences of concepts underlying AGG and EMF TIGER}

The conceptual differences between both tools lie in the fact that AGG is a graph transformation environment for typed, attributed graphs, so the concepts of object-based modeling are captured here by the notions of graphs (nodes and edges for objects and relations), type graphs, and attributes. On the contrary, EMF TIGER manipulates EMF instance model directly (in-place) using EMF model manipulation methods. For an overview, Table 1 shows a comparison of main EMF notions to their counterparts in graph terminology.

Classes in an EMF model correspond to nodes in a typing graph. Associations between classes can be seen as edges in a type graph. Generalizations and multiplicity constraints of association ends can also be defined in the type graph. Objects as instantiations of classes of an EMF model are comparable to nodes in a graph which is typed by a type graph. Objects can be linked to each other by setting reference values. Such references correspond to edges in a typed attributed graph. EMF models always have an inversed reference for each reference. Since EMF TIGER works directly on EMF instance models and generates new references by

\footnotetext{
${ }^{2}$ Since November 2009, the tool is also available under the name EMF Henshin as ECLIPSE subproject of the ECLIPSE Modeling Framework Technology project EMFT, see http://www.eclipse.org/modeling/emft/ henshin.
} 
Table 1 Mapping EMF notions to graph terminology

\begin{tabular}{ll}
\hline EMF notion & Graph terminology \\
\hline Model & $\begin{array}{c}\text { Type graph } T G . \text { Edges can be marked } \\
\text { as containments. }\end{array}$ \\
Inheritance & $\begin{array}{l}\text { Node type inheritance in } T G \\
\text { Class }\end{array}$ \\
Node in type graph $T G$ \\
Association & Edge in type graph $T G$ \\
Multiplicities & Node and edge type multiplicities in $T G$ \\
Class attributes & Attribute types belonging to node types \\
Model instance & $T G$-typed, attributed graph $G$ with containment \\
& $\quad$ edges and typing morphism $G \rightarrow T G$ \\
Object & Node in $T G$-typed graph $G$ \\
Reference & Edge in $T G$-typed graph $G$ that must not violate \\
& certain multiplicity and containment constraints \\
\hline
\end{tabular}

calling the respective EMF method, it is ensured that corresponding inverse references are generated as well. Note that the EMF concept Package does not exist in graph terminology. Since EMF transformation rules in EMF TIGER only transform EMF instance models, packages are not used in rules. Moreover, EMF models have containment constraints in addition, which do not occur in typed graph transformation. In [5], containment constraints of EMF model transformations are translated to a special kind of EMF model transformation rules such that their application leads to consistent transformation results only. Thus, we can identify a kind of EMF model transformations which behave like typed graph transformations. As a consequence, the theory of algebraic graph transformation becomes applicable to these EMF model transformations to show functional behavior and correctness properties.

\section{Solutions}

\subsection{Assignment 1: conference scheduling}

In this section, we describe the solution of the conference scheduling problem. In Sect. 4.1.1, we present the underlying EMF model and AGG type graph. For the solution of Assignment 1, we present the rules (modeled in AGG and EMF TIGER) for automatic scheduling the available papers into time slots such that certain constraints are fulfilled. These constraints are: no person is at two places at a time, and each session uses consecutive time slots (Sect. 4.1.2). Moreover, we use the concept of graph constraints in AGG to check conditions on graphs (Sect. 4.1.3) and generate rule code in EMF TIGER to be used in Java applications (Sect. 4.1.4). In Sect. 4.1.5, we ensure that there are no unused time slots between used ones.

\subsubsection{The meta model}

Figure 1 shows the EMF model for the Conference Scheduling System which is used in EMF TIGER.

A Conference contains Persons, Presentations, Sessions and Slots. A Person holds one or more Presentations and chairs one or more Sessions. Presentations are in a Session and scheduled in Slots, which are linked as a list by next arcs. Slots are used by Sessions.

The AGG type graph for the Conference Scheduling model is shown in Fig. 2. It is similar to the EMF model in Fig. 1, with the difference that type graphs in AGG do not have container types and containment edges. Multiplicities for arc type ends can be used like in EMF. For attribute types, default values can be defined which are assigned to new instance nodes of that type (e.g. the value for used is set to false in a new Slot node by default). Note that the Slot attribute used has a different meaning than the edge of type "used". The boolean attribute denotes whether the slot is used at all, whereas the "used"-edges give the concrete session scheduled to the slot (see Sect. 4.3.2 where the boolean attribute is needed).

Generalization and inheritance arcs can be used also in AGG, as shown for the named node types. Moreover, in con-

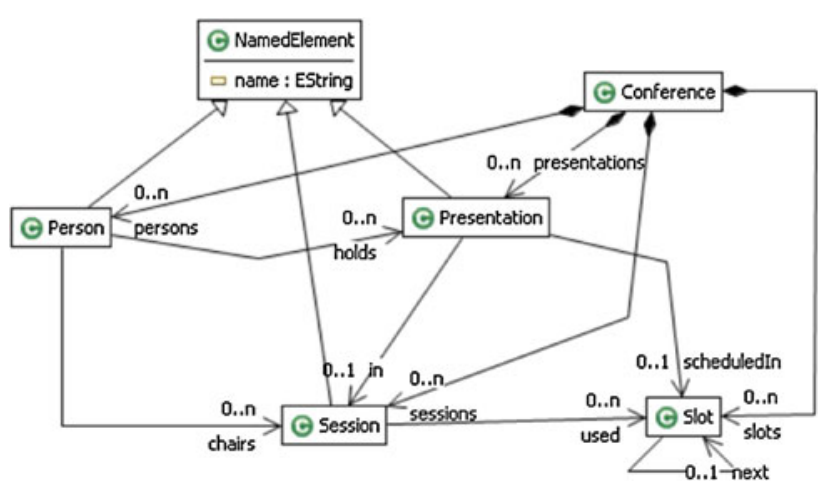

Fig. 1 EMF model for Conference Scheduling in EMF TIGER

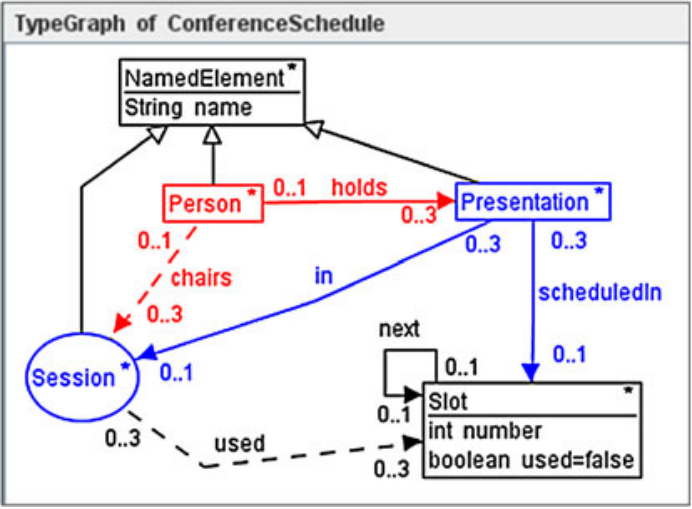

Fig. 2 Type graph for Conference Scheduling in AGG 


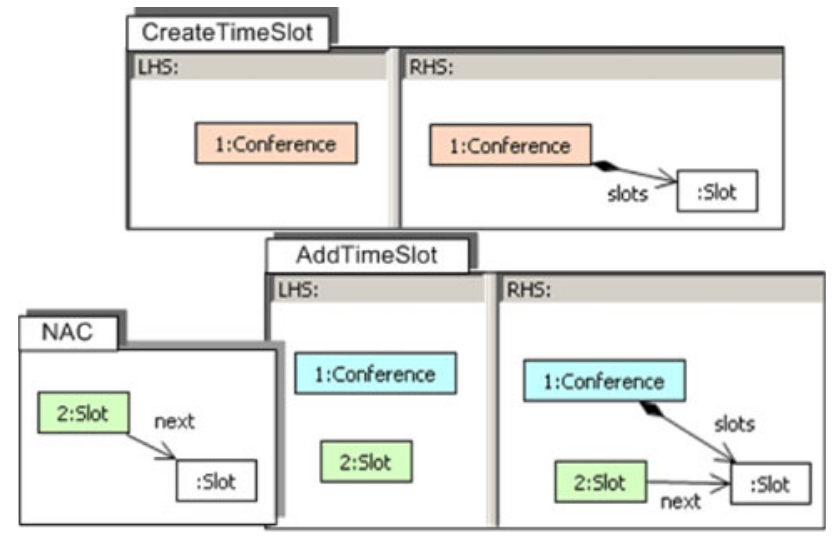

Fig. 3 Rules CreateTimeSlot and AddTimeSlot

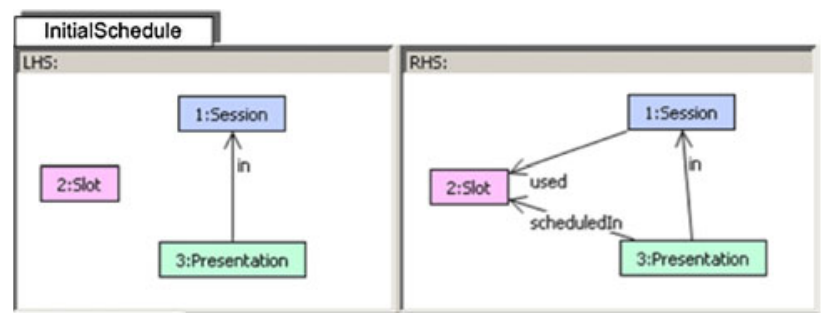

Fig. 4 Rule InitialSchedule

trast to EMF TIGER, we can have individual multiplicities for nodes in AGG.

\subsubsection{Rules for conference scheduling}

The rules presented in this section are shown as screenshots from EMF TIGER. In principle, the graph rules for conference scheduling in AGG are very similar, hence we do not depict them here, too.

Figure 3 shows two rules which create and link time slots. Initially, the first time slot is created (rule CreateTimeSlot). If slots exist, a new slot is appended to the currently last slot by applying rule AddTimeSlot.

The scheduling algorithm has quite simple rules but a lot of conditions that control their application. These conditions are formulated as NACs (negative application conditions) of the respective rules. Rule InitialSchedule, shown in Fig. 4, models the initial operation which schedules a presentation and a session into a time slot for the first time.

Eight NACs (depicted in Fig. 5) control the rule application by preventing that the rule is applied in one of the following cases:

1. The presentation to be scheduled is already scheduled in another time slot.

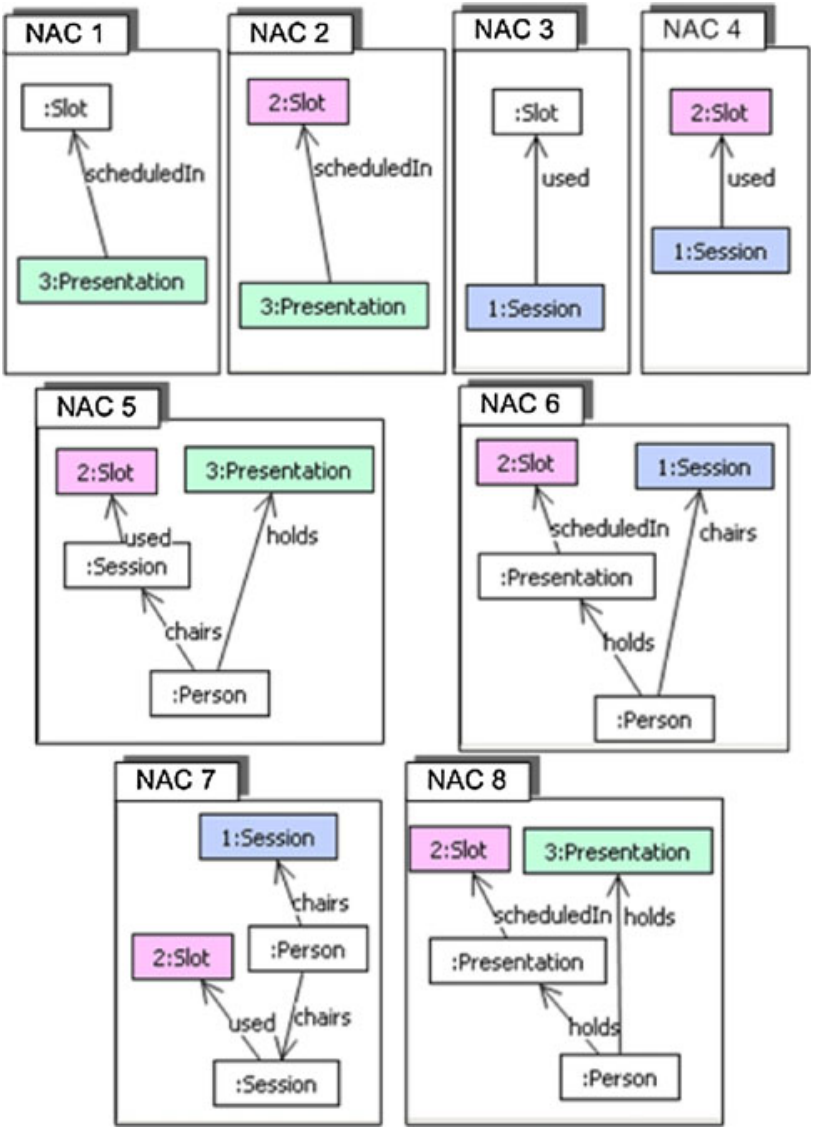

Fig. 5 NACs for Rule InitialSchedule

2. The presentation to be scheduled is already scheduled in the time slot where it is supposed to be scheduled in by the rule. ${ }^{3}$

3. The session already uses a different time slot (in this case, one of the two other scheduling rules will be used to schedule the presentation next to one of the same session).

4. The session already uses the time slot where the presentation is supposed to be scheduled in.

5. The presenter of the presentation to be scheduled chairs a session which uses the same time slot.

6. The session chair holds a presentation scheduled in the same time slot.

7. The person who is supposed to be session chair, chairs already another session scheduled in the same time slot.

8. The person who is supposed to be presenter, presents already another paper scheduled in the same time slot.

The next two rules ScheduleAfter and ScheduleBefore, shown in Fig. 6, take care of grouping papers of one session into consecutive time slots, i.e. a presentation is scheduled

$\overline{3}$ This condition is not covered by NAC 1 because we allow for injective matches, only. 


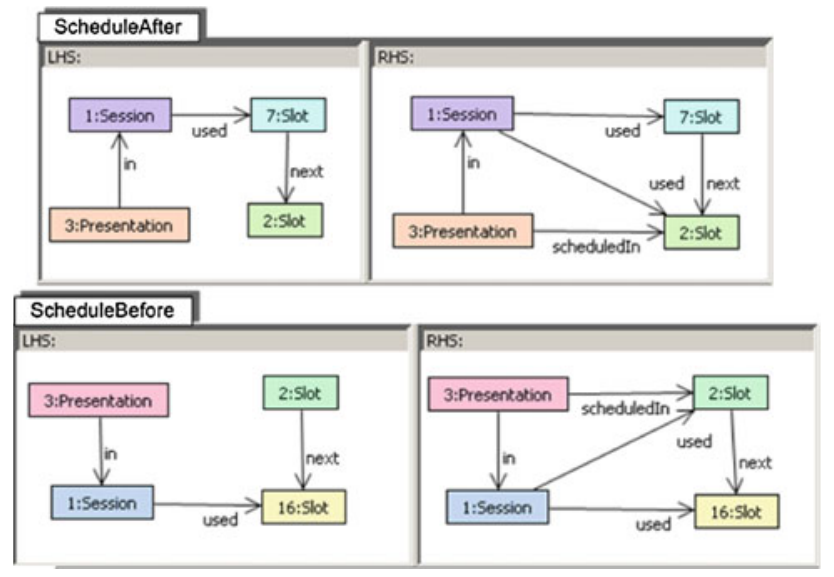

Fig. 6 Rules ScheduleAfter and ScheduleBefore

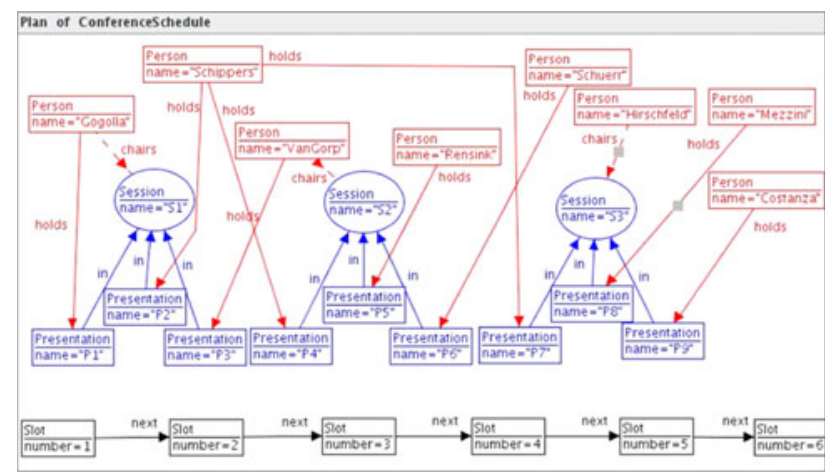

Fig. 7 Start graph Conference Plan

in a free time slot either directly before or after a slot where there is already a scheduled paper of the same session.

The NACs of both rules in Fig. 6 are analogous to the NACs of rule InitialSchedule in Fig. 5, with the slight difference that we do not need NAC 3.

\subsubsection{Model debugging in AGG}

In contrast to EMF TIGER, AGG has a visual view for instance graphs, such that rule applications can be tested and debugged visually. Figure 7 shows the start graph for the scheduling which is typed over the type graph in Fig. 2 and corresponds to the conference plan of the case study given in [25].

Applying the three scheduling rules to the start graph as long as possible leads to corresponding changes of the start graph in-place, i.e. the stepwise changes can be observed in the instance graph view. A possible result is depicted in Fig. 8, where edges of type used and of type scheduledIn are now linking the sessions and presentations to time slots.

For big and complex graphs with many nodes and arcs, properties may not be easy to discern. AGG allows the modeler to define graph constraints which can be checked after

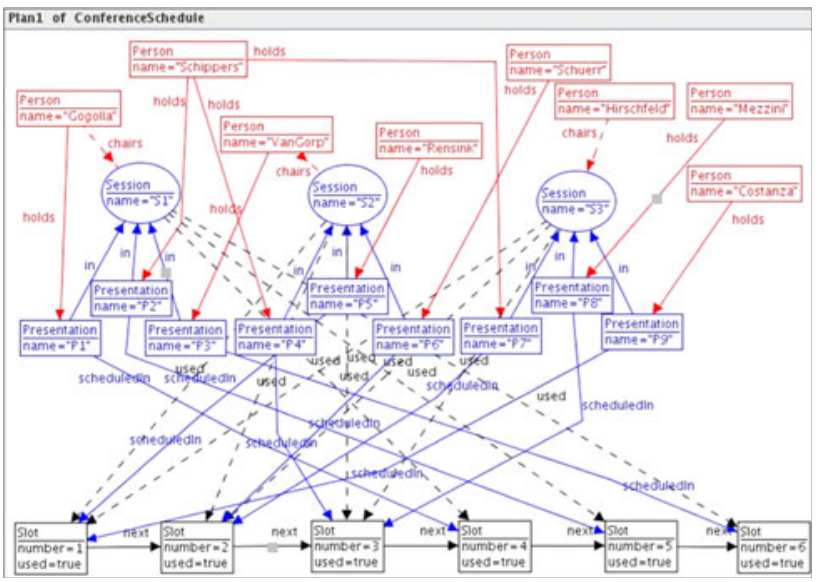

Fig. 8 Graph after scheduling is completed

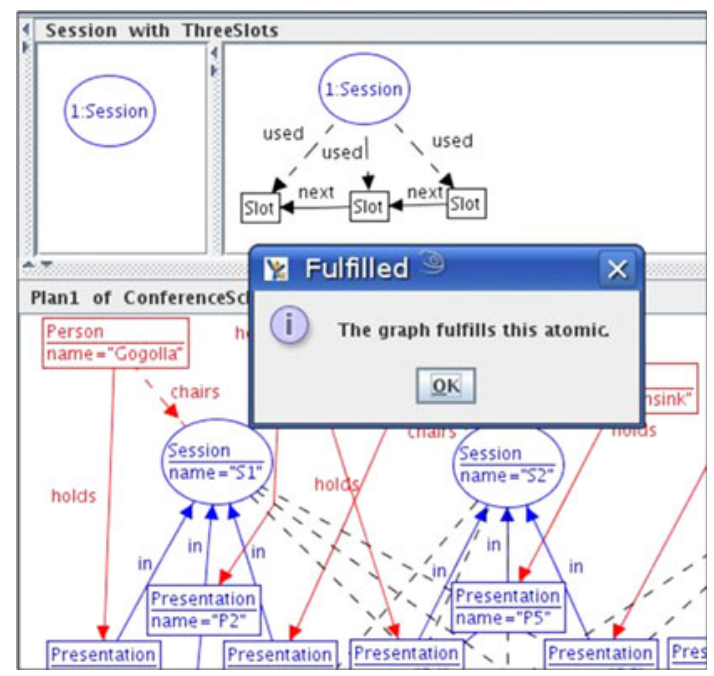

Fig. 9 Checking the atomic graph constraint ThreeSlots

transformations (see Sect. 2). It is possible to let an automatic transformation stop when a constraint is fulfilled or violated.

Figure 9 shows an atomic graph constraint in the top row which defines the constraint that a session is always scheduled in three consecutive slots. Checking this constraint on the graph in Fig. 8 leads to the popup message "The graph fulfills this atomic" as shown in Fig. 9.

\subsubsection{Generated Java code in EMF TIGER}

From the scheduling rules defined in the last section, $E M F$ Tiger generates Java code. Figure 10 shows the generated execute() method for rule CreateTimeSlot from Fig. 3. An execute() method is generated for each rule. At first, a helper structure (called wrapper) is created, where input parameters are assigned to the corresponding model elements. Then, a match is computed by calling the wrapper method getSolution(). At last, if a match is found, the rule is applied by 


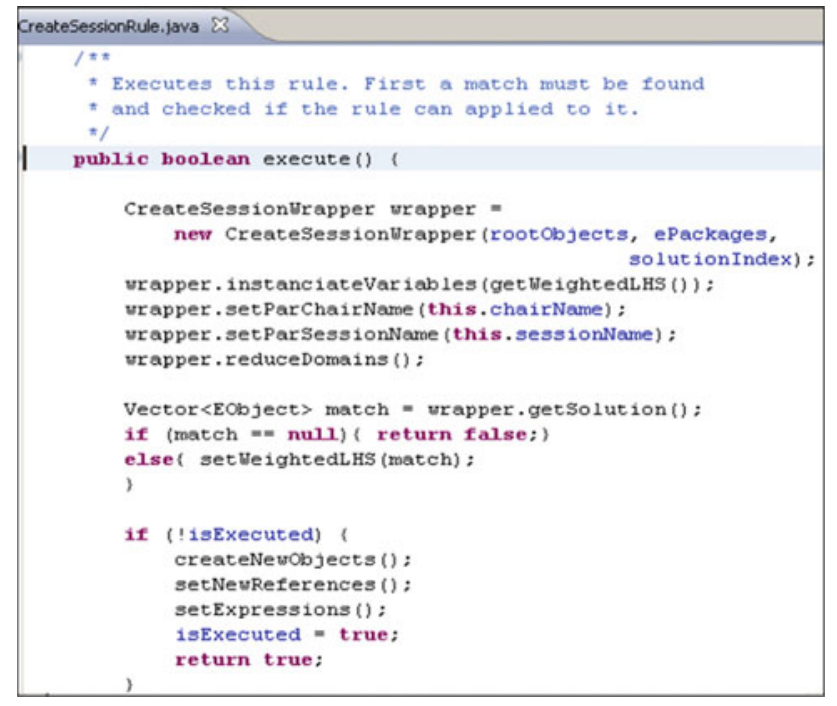

Fig. 10 Generated execute()-method for rule CreateSession

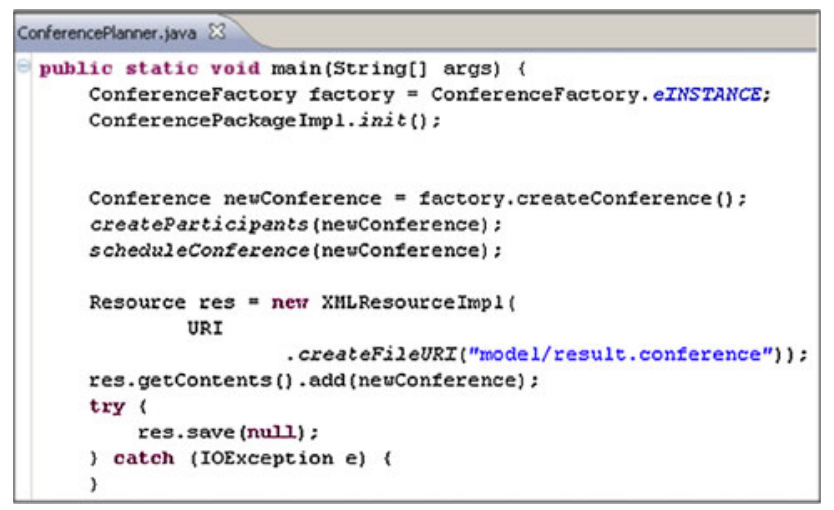

Fig. 11 Main method of the Conference Planner application

creating all new objects and setting all new references and attribute expressions in the EMF instance, which is thus transformed in-place. (For rules where also objects are deleted, a corresponding deletion method is generated, as well.)

The generated rule code is used in an application as shown in Fig. 11: a ConferenceFactory instance is used to create a new Conference (named newConference). Rule applications can now be evoked for newConference, which is done in the two methods createParticipants() and scheduleConference() (explained below). Since EMF TIGER does not yet have a visual debugger, where the results of rule applications can be shown as graphs, we create a text file "result.conference" where we will save the resulting model instance in order to test our system.

In our example, a rule application sequence is contained in method createParticipants(), where instance creation rules (explained later in Sect. 4.2.1) are applied "by hand" to create papers, presenters, sessions and session chairs. Parts from this sequence which produces the instance model from [25],

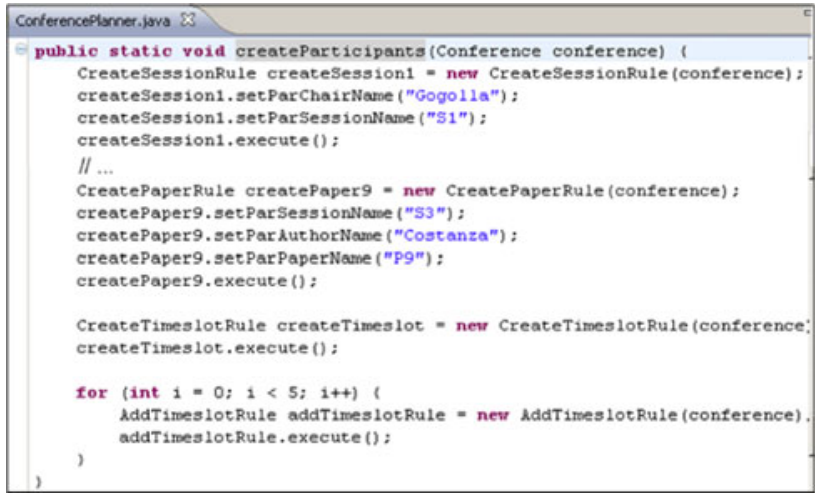

Fig. 12 Method createParticipants

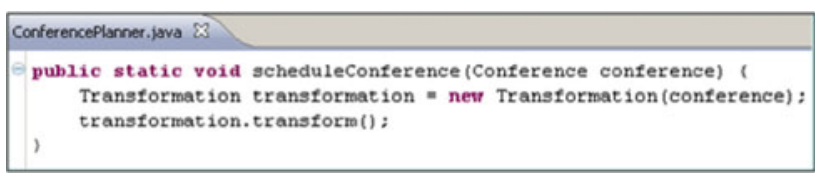

Fig. 13 Method scheduleConference

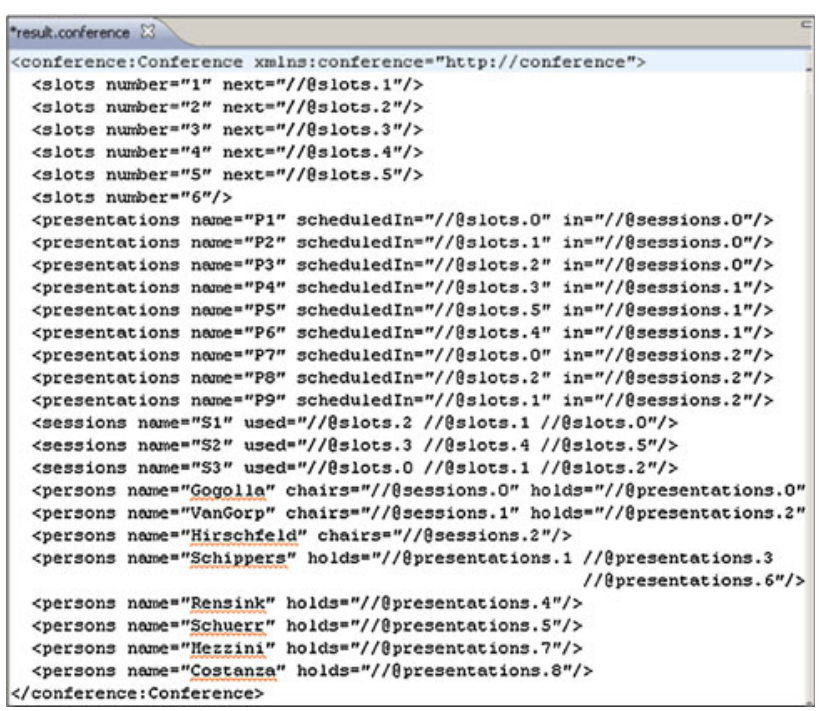

Fig. 14 One resulting solution after automatic scheduling

are listed in Fig. 12. In addition, six time slots are created in a for-loop.

In contrast to listing the applied rules explicitly, method scheduleConference() calls a general transform() method where arbitrary rules inside a given layer are applied at arbitrary matches. The rule application continues until no more rule of the current layer is applicable. This automatic transformation realizes the scheduling of papers into time slots by applying the rules from Sect. 4.1.2. The code of the method scheduleConference() is shown in Fig. 13.

After the automatic scheduling has been finished, the model instance is documented in file result.conference, shown in Fig. 14. 


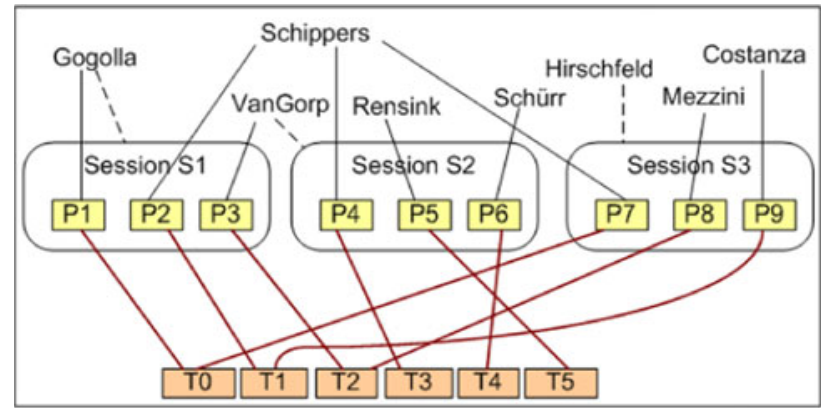

Fig. 15 Resulting solution drawn as graph

In most cases, the file contains one of the possible valid solution of the scheduling problem. Sometimes, not all papers could be scheduled in a consistent way due to the arbitrary choice of a time slot for the first papers. Then, the result file contains unscheduled papers. To get a full valid solution, the scheduling algorithm should be repeated until the computed solution contains only scheduled papers. The validity of our sample solution can be verified best by drawing the model instance from Fig. 14 as graph, as shown in Fig. 15. It can be easily seen that no person is at two places at a time and that papers within one session are scheduled in consecutive time slots. ${ }^{4}$

\subsubsection{Taking care of unused time slots}

An additional subtask of the scheduling problem is to ensure that unused time slots are not placed between used time slots, i.e. they can be found in the beginning or in the end of a conference, only. For our example (see Fig. 15), this problem can arise if we allow more than six time slots for scheduling.

Being able to control rule applications in EMF TIGER, we solve this problem as follows: since unused slots are not linked to any session, we may remove them from the queue, i.e. we delete all unused slots which have at least one predecessor and one successor slot, counting the deletion steps. After deletion is finished, we add as many slots arbitrarily either at the beginning or at the end of the time slot chain. Thus, in the end we have as many slots as before but they are situated only at the beginning or at the end of the conference time table.

The rule for slot deletion is shown in Fig. 16. Two NACs ensure that the slot is unused by sessions and not scheduled for any presentation.

Two rules, shown in Fig. 17, are provided to add unused slots either at the start of the time slot chain (rule AddSlotAtStart) or at its end (rule AddSlotAtEnd).

\footnotetext{
4 This is a simple test-and-set algorithm. But it would also be possible to implement an algorithm where non-deterministic choices have to be thrown away, once they do not result in a valid solution.
}

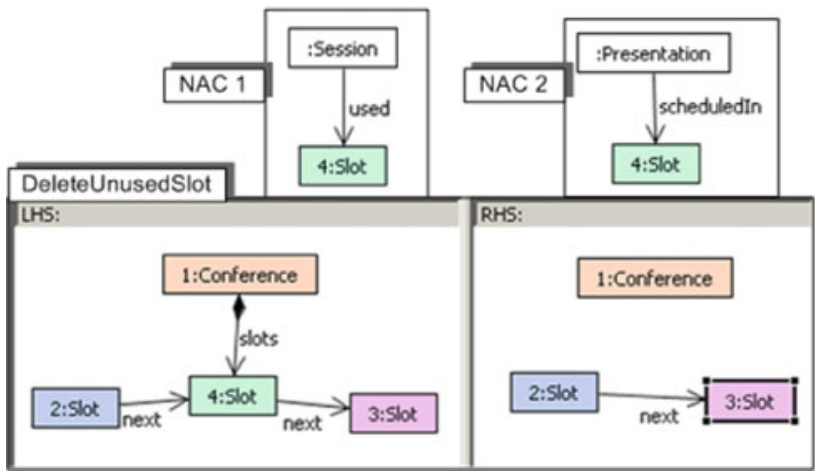

Fig. 16 Rule DeleteUnusedSlot

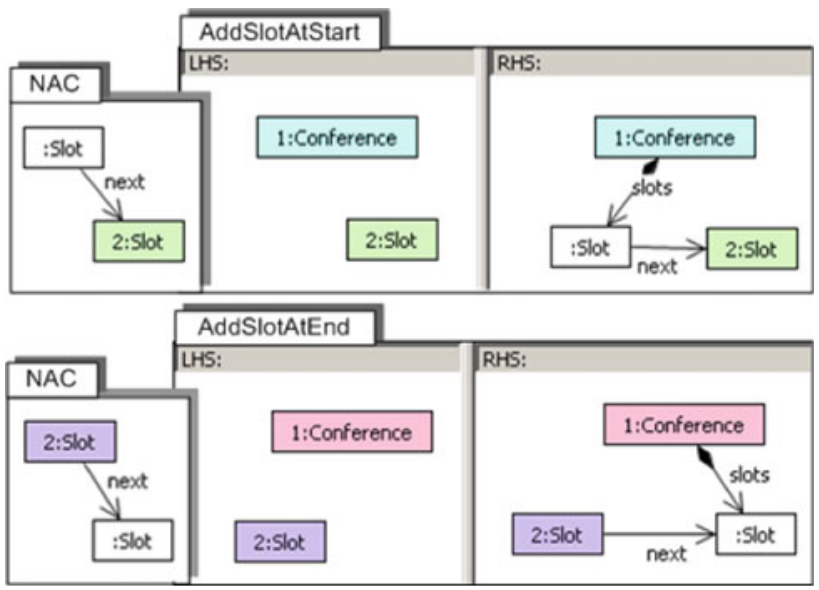

Fig. 17 Rules AddSlotAtStart and AddSlotAtEnd

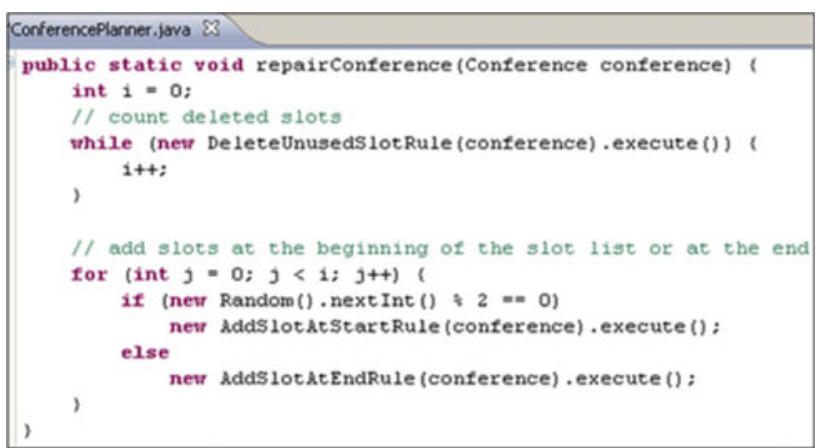

Fig. 18 Java code to control re-allocation of unused time slots

The rule application is controlled by Java code, as shown in Fig. 18. Counter $i$ counts the deleted slots by counting how many times rule DeleteUnusedSlot is applied. The for-loop then applies one of the two rules AddSlotAtStart or AddSlotA$t E n d$ at random, as many times as there have been deletions before.

A different strategy for finding unused time slots is followed in AGG, where controlled rule application is limited. Here, we add a graph constraint UsedSlots which checks whether there are unused time slots between used ones. We 


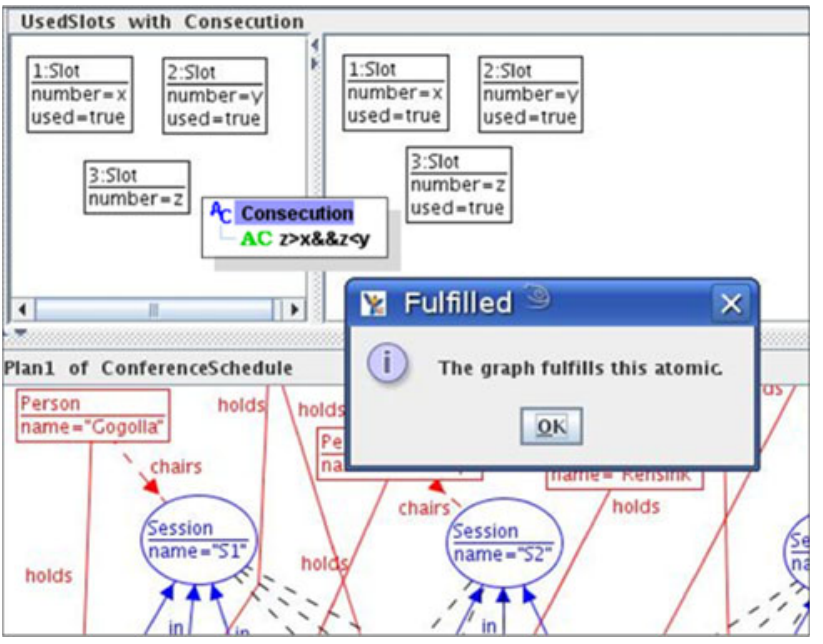

Fig. 19 Graph constraint UsedSlots

have to take care that also unused time slots are found which have no direct used neighbor slots. Therefore, the values of the number attributes of slots are compared, and the constraint (depicted in Fig. 19) requires that whenever the number of a time slot (denoted by variable $z$ ) lies somewhere between the numbers of two used time slots ( $x$ and $y$ ), then the time slot $z$ must be a used one, too.

The comparison of slot numbers is formulated by the attribute condition $\mathrm{z}>\mathrm{x} \& \& \mathrm{z}<\mathrm{y}$ of the graph constraint. If this constraint is valid for all time slot combinations, then there are no empty time slots between used ones. Of course, this constraint has to be checked after the scheduling has been finished, and not after every step of the algorithm. Therefore, for rule application control in AGG, we use layered graph transformation with the additional possibility to reset and restart the transformation again with the original start graph if a constraint is not satisfied (we make use of the transformation options loop over layers, reset graph before loop and consistent at the end). Hence, AGG computes solutions until a solution is found which satisfies the constraint, i.e. which has no unused time slots in the middle of the schedule. Checking the constraint UsedSlots on the graph in Fig. 8 yields that it is fulfilled.

As an alternative to computing new solutions from the scratch in the case that the constraint UsedSlots is not fulfilled, we offer an additional rule, called ChangeSchedule which can be applied manually. It adapts the computed schedule in a way respecting the condition that no person must be at two places at a time. Hence, this rule (shown in Fig. 20) has six NACs analogous to the NACs in Fig. 5, checking that all of our basic scheduling constraints are fulfilled. If, in addition, the constraints UsedSlots (Fig. 19) and ThreeSlots (Fig. 9) are fulfilled, we have found a solution which satisfies all requirements stated by assignment 1 (see Sect. 4.1).

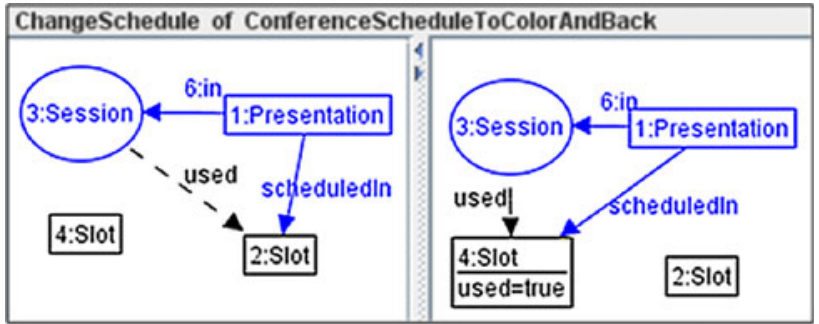

Fig. 20 Rule ChangeSchedule

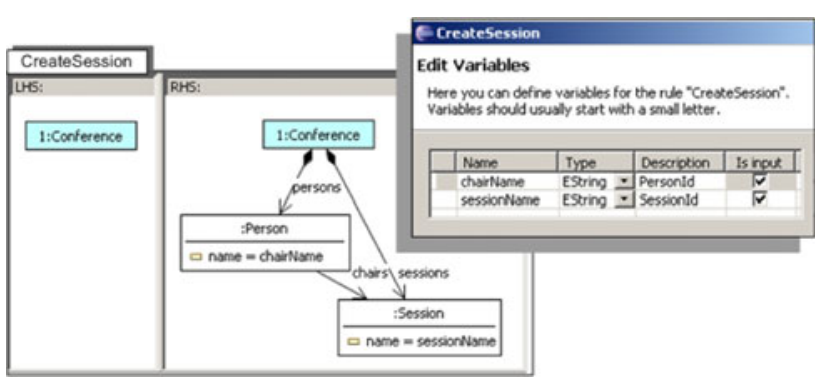

Fig. 21 Rule CreateSession

\subsection{Assignment 2: instance generation}

This task is to generate all possible instances of presentations, sessions, persons and slots with adaptable restrictions, such as

- there are exactly five persons,

- there are exactly three sessions,

- there are exactly six slots,

- there are exactly three papers per session,

- at least one person is chair and presents two papers,

- at least one person is presenting in two different sessions.

Using our tool EMF Tiger, we present the ability of the tool to add Java code in order to control the application of rules, e.g. by adding conditions and loops.

\subsubsection{Rules to create conference participants}

In order to build a conference instance model, we have formulated a few rules. Figure 21 shows rule CreateSession which adds a session and its chair to the system. The variable view window shows that the name attributes of the newly created Person and Session elements are of type String and are input variables, e.g. they have to be defined interactively when the rule is applied.

Figure 22 shows three rules CreatePaper, CreatePerson and LinkPersonPaper which create presentations and their presenters. Either its presenter is created together with the presentation (rule CreatePaper), or the presenter is created separately (rule CreatePerson) and has to be linked to an 


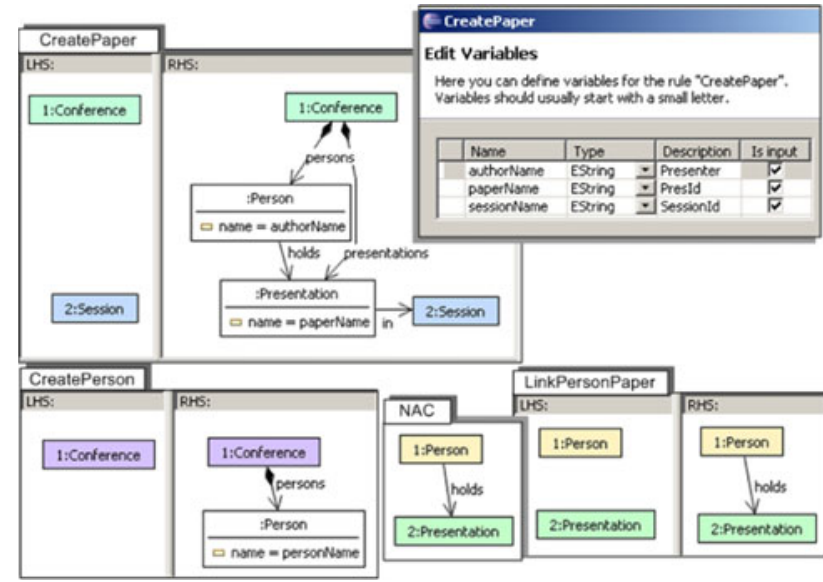

Fig. 22 Rules CreatePaper, CreatePerson and LinkPersonPaper

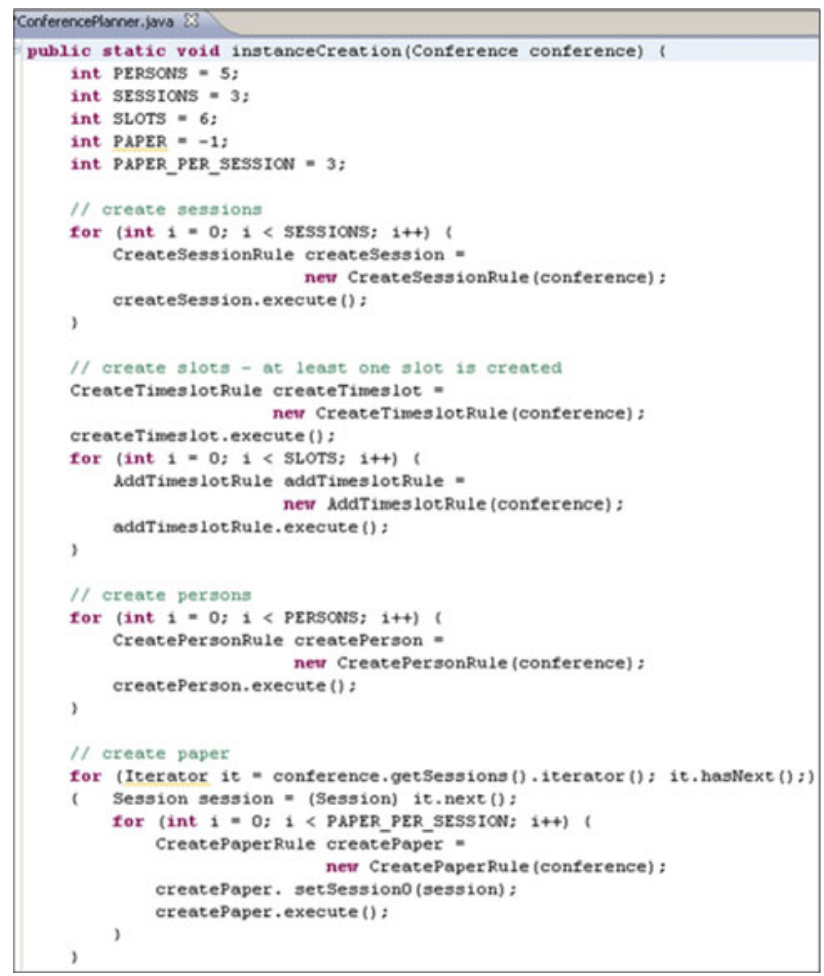

Fig. 23 Java code for instance generation

existing presentation (rule LinkPersonPaper). An analogous rule exists to link a person to a session.

In order to control the instance generation, loops may be defined in the Java application code which count the generated model objects. Figure 23 shows the Java method instanceCreation which generates the instances for this assignment, as given in Sect. 4.2: the first three loops generate three sessions, six time slots and five persons. In the last loop, for each session there are created three papers.

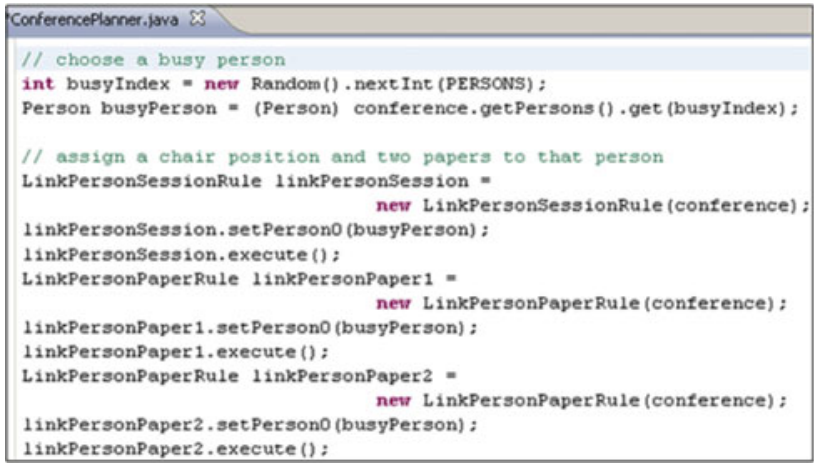

Fig. 24 Java code for generating a chair presenting two papers

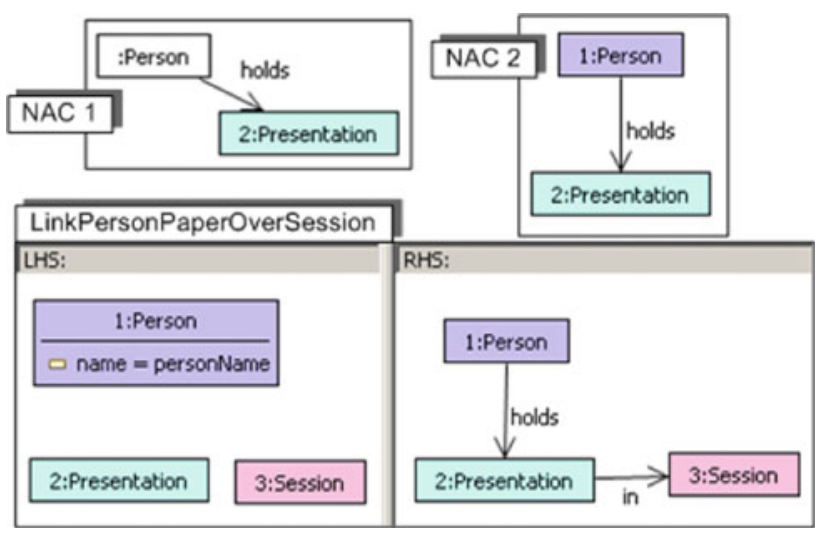

Fig. 25 Rule LinkPersonPaperOverSession

The code in Fig. 24 selects a person at random and links him to a session by applying rule LinkPersonSession and to two papers by applying rule LinkPersonPaper twice.

For the last task, the generation of a person who is presenting in two different sessions, we need one additional rule LinkPersonPaperOverSession (see Fig. 25) which links a person to a presentation and the presentation to a session.

The control code in Fig. 26 now applies this rule twice to the same person but to two different sessions.

\subsection{Assignment 6: interoperability}

This task is to demonstrate interoperability of the tool. We implemented this assignment using AGG by refactoring the conflict resolution into three steps:

1. Mapping the instance model to the domain of colored graphs.

2. Computing a coloring, (alternatively by a particular graph grammar or by reusing a 3GL library for computing a minimal coloring).

3. Mapping the computed solution back to the time slot domain (by graph grammar). 


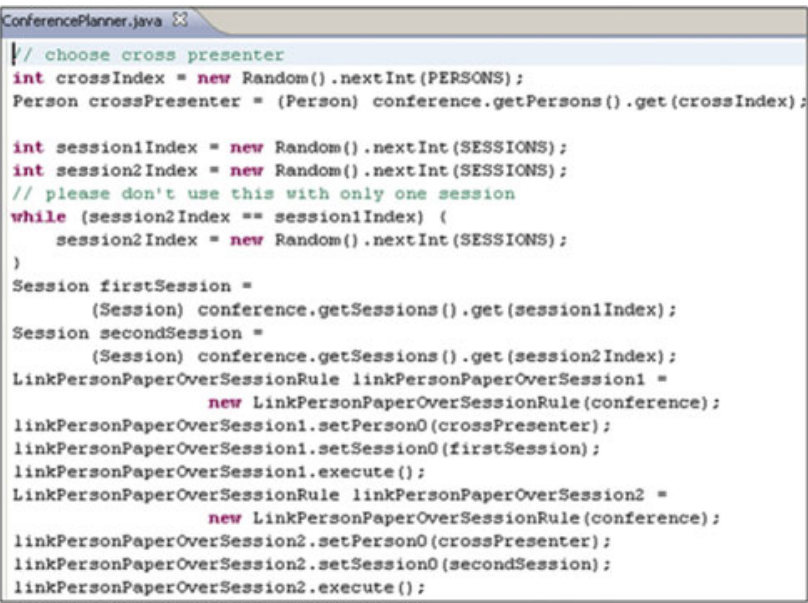

Fig. 26 Java code for generating a presenter in two sessions

At first, we describe the model transformation Conference2Color from the conference scheduling domain to the domain of colored graphs. We then explain our simple coloring algorithm in AGG for computing node colors such that no node has the same color as one of his neighbors. Alternatively, we have implemented an extension in AGG to export a graph to the DIMACS format [9] in order to apply an external minimal-coloring algorithm to it. After the application of the external tool, AGG can import the solution in order to color the graph correspondingly. In the last step, a backward model transformation is realized transforming the colored graph to the conference scheduling domain.

\subsubsection{Model transformation to colored graphs}

For the model transformation Conference2Color, the conference schedule type graph is extended (see Fig. 27) by a new node type Node which corresponds to the node type Presentation (the correspondence is modeled by the Node attribute name which is supposed to be set to the value of the corresponding Presentation name attribute). Type Node has a color attribute (a number), and a new edge type connecting two neighbor Nodes.

The model transformation rules are depicted in Fig. 28. Rule Presentation2Node generates a node for each presentation. The remaining rules find pairs of presentations that cannot be scheduled in parallel, i.e. they check for one of the following situations:

1. two papers are presented by one person,

2. two papers are in different sessions with the presenter of one paper being the chair of the other paper's session,

3. two papers are in different sessions with the same chair,

4. two papers are presented by one person in the same session.

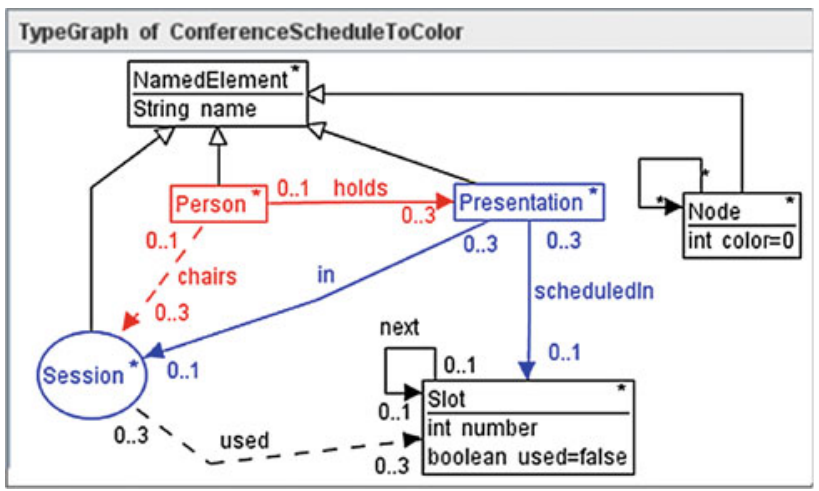

Fig. 27 Extended type graph for model transformation

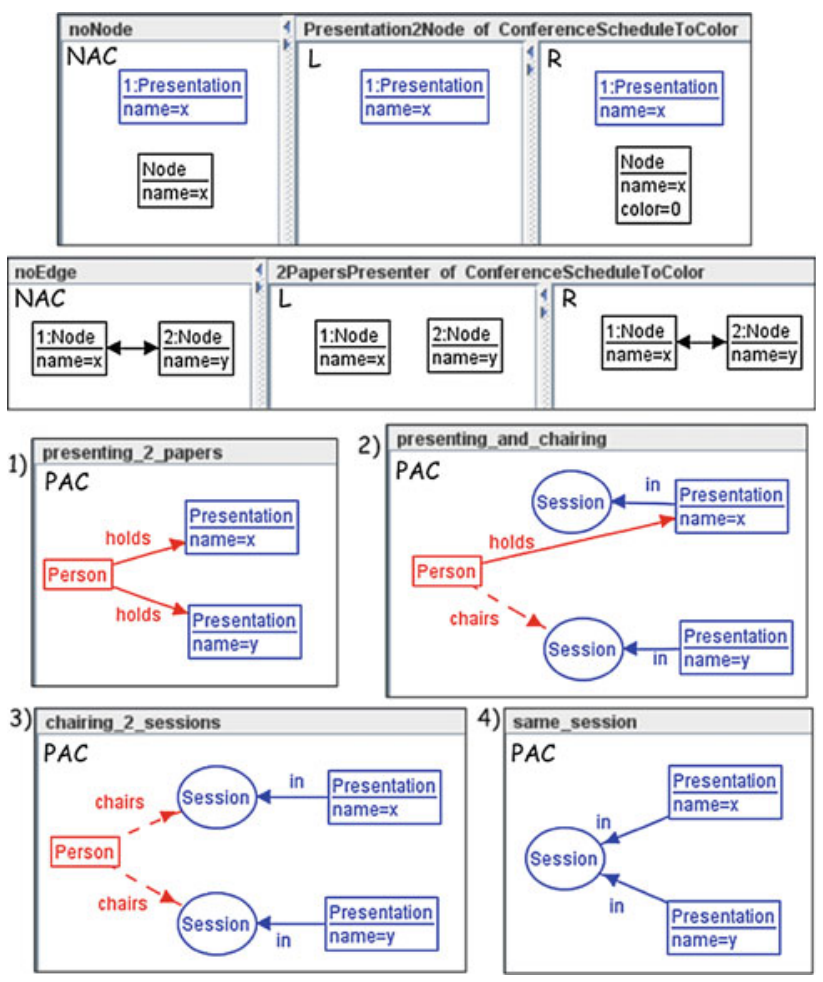

Fig. 28 Model transformation to the domain of colored graphs

All four pair-finding rules add a bidirectional edge ${ }^{5}$ between two nodes corresponding to presentations that cannot be scheduled in parallel. Each such rule has a NAC forbidding the existence of a bidirectional edge between the two nodes involved. Hence, all four rules look like rule $2 \mathrm{~Pa}$ persPresenter in Fig. 28. The difference of the pair-finding rules has been modeled by four different positive application conditions (PACs). In contrast to a NAC, a host graph is checked for the existence of the graph structure modeled by the PAC, and the rule can be applied only if the

$\overline{5}$ In AGG, bidirectional edges correspond to two edges in opposite directions. 


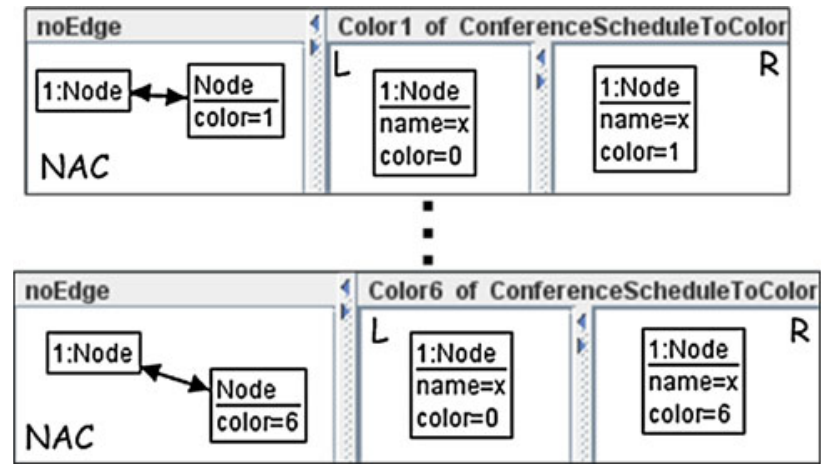

Fig. 29 Rules for the coloring algorithm

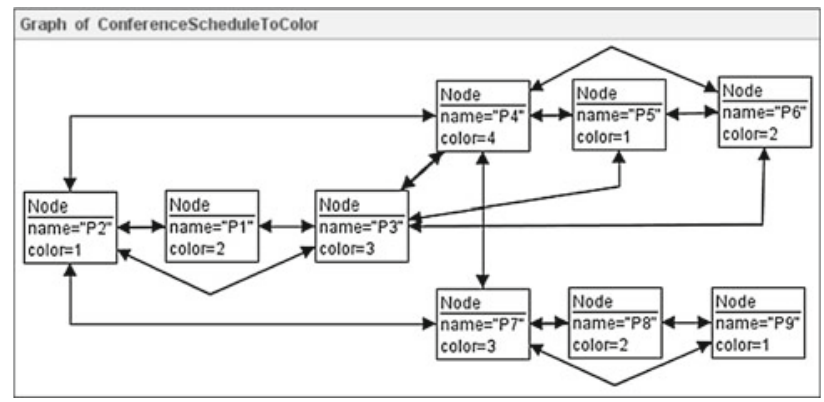

Fig. 30 Result after applying the coloring algorithm

PAC is present in the graph. PACs are a valuable means to make a rule more concise, because context graph items do not have to be drawn in the left-hand and in the right-hand side when they are not used directly for the changes modeled by the rule. In Fig. 28, the four PACs are numbered according to the enumeration of critical situations given above.

\subsubsection{The coloring algorithm implemented in AGG}

In order to realize a valid coloring, i.e. a coloring of nodes such that no node has the same color as one of its neighbors, we implemented a simple coloring algorithm in AGG by defining Rules Color1-Color6 (assigned to separate layers), shown in Fig. 29.

The coloring rules all work according to the same principle: in each layer, all uncolored nodes (with color attribute being 0 ) get assigned color $=i$ if they do not have a neighbor node of that color. This is repeated as long as possible. Then, $i$ is set to $i+1$, and the procedure is repeated in the next layer with the next color. We provide 6 different colors, because the start graph has six time slots, i.e. we have six coloring rules in different layers. One computed coloring result is shown in Fig. 30. All nodes belonging to the same session triple (P1,P2,P3), (P4,P5,P6) or $(\mathrm{P} 7, \mathrm{P} 8, \mathrm{P} 9)$ are connected to each of the other session triple member. Moreover, there are edges connecting P4 and P2 (resp. P2 and P7, resp.

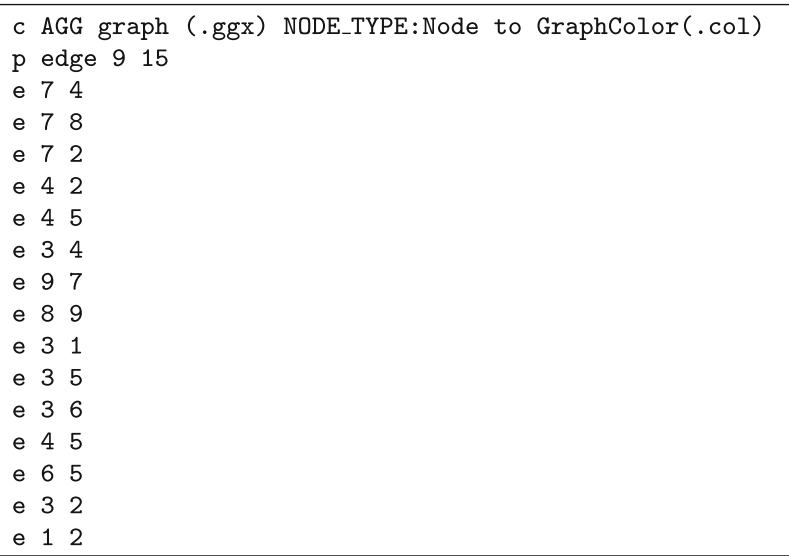

Fig. 31 Input file for the Greedy algorithm

$\mathrm{P} 4$ and P7) as these presentations have the same presenter (see Fig. 15), and there are edges connecting P3 with P4, P5, $\mathrm{P} 6$ (Van Gorp is giving presentation $\mathrm{P} 3$ and chairing the session with P4, P5, P6). Obviously, in the computed coloring, no two neighbors have the same color. Note that we need at least four colors for a valid coloring since P4, P5, P6 always need three different colors as they are in the same session, and P3 must have yet another color because it represents a presentation of the session chair in a different session.

\subsubsection{Interoperability: linking AGG to the external Greedy algorithm}

As alternative solution of the coloring problem which even guarantees a minimal coloring, we export our graph (the subgraph after the model transformation which contains only nodes of type Node and the bidirectional edges connecting them) to the DIMACS format which can be understood by the program Greedy implementing a minimal graph coloring algorithm $[8,9]$.

The export facilities of AGG have been extended by the option Export to ColorGraph which takes the graph consisting of Nodes and their adjacent edges and stores it in the text format DIMACS [9] required by the Greedy tool. The listing in Fig. 31 shows that basically the edges are exported, where the source and target nodes are given by numbers. ${ }^{6}$

The coloring is now computed externally, by applying the Greedy algorithm [9] on the input file in Fig. 31. The greedy algorithm, also known as the sequential algorithm, takes each node in turn in some predefined order and tries to color the vertex with one of the colors used so far. In other words, it tries to add the vertex to one of the existing color classes. If it is not possible to color it with any existing color, then a new color class is created and the vertex is assigned the color

\footnotetext{
6 This always defines the complete color graph since we do not have isolated nodes.
} 
CLRS 4 FROM GREEDY $\mathrm{cpu}=0.00$ pid $=22166$

$\begin{array}{lllllllll}1 & 2 & 3 & 4 & 1 & 2 & 3 & 1 & 2\end{array}$

Fig. 32 Output file from the Greedy algorithm

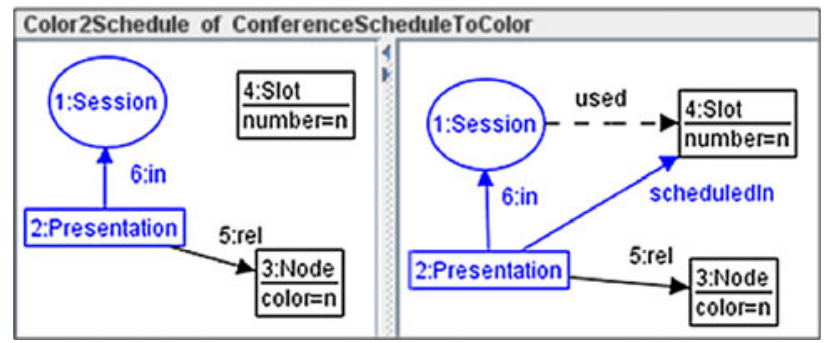

Fig. 33 Rule Color2Schedule

of that class. The result of the algorithm is again stored into a file (see Fig. 32). The numbers correspond to colors, and their order corresponds to the node numbering.

AGG has been extended by an option to import a coloring, which translates the result from the DIMACS output file back by setting the color attributes of the respective nodes to the color numbers in the file. The result is a graph similar to the result in Fig. 30 after applying the coloring rules.

\subsubsection{Backward model transformation Color2Schedule}

After the coloring has been computed by AGG using the coloring rules from Sect. 4.3.2, or, alternatively, the output file from Greedy has been imported back to AGG, the coloring result has to be transformed back to the conference scheduling graph. Rule Color2Schedule, shown in Fig. 33, realizes this backward transformation. ${ }^{7}$ Each color number of a node corresponds to the number of the time slot for the node's presentation. So, no conflicting presentations are scheduled to the same time slot.

Please note that the resulting coloring may not yet respect the constraints that all presentations within a session should be scheduled in three consecutive slots, and that there must not be unused slots between used ones. In order to satisfy these constraints, the corresponding graph constraints shown in Figs. 9 and 19 have to be checked in the end. For the internal coloring solution, a loop over layer may be performed (i.e. the coloring procedure is repeated) if one of the constraints is not valid.

Alternatively, rule ChangeSchedule (see Fig. 20) may be applied manually, adapting the computed solution until all graph constraints are valid.

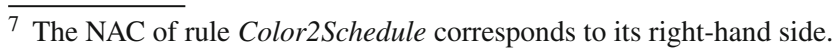

\subsection{Assignment 3: property verification}

In this section, we demonstrate the verification tools of AGG in order to verify properties of the model transformation from the conference scheduling domain to the domain of colored graphs presented in Sect. 4.3.1. We want to show that this model transformation is functional, i.e. it is terminating and the result we get is always unique for each input model.

The termination check implements the algorithm described in [12]. In general, termination is undecidable for graph transformation systems. But if a system meets certain termination criteria, we can conclude that it is terminating. The proof for termination of graph transformation systems [12] is based on layered graph transformation systems with deletion and non-deletion layers. Informally, the deletion layer conditions express that the last creation of a node of a certain type should precede the first deletion of a node of the same type. Furthermore, each rule application should delete more items of a certain type than it creates. Non-deletion layer conditions ensure that if an element of a certain type occurs in the LHS of a rule then all elements of the same type were already created in previous layers.

Figure 34 shows the automatically computed deletion and non-deletion layers for our Conference2Color model transformation. The termination conditions are fulfilled by the set of model transformation rules from Fig. 28.

The second condition for functionality is confluence of the model transformation. The graph transformation theory allows us to compute conflicts and dependencies of transformations by relying on the idea of critical pair analysis. AGG provides an algorithm implementing this analysis. Critical pair analysis is known from term rewriting and can be used to check if a rewriting system can contain conflicting computations. Critical pairs formalize the idea of showing a conflicting situation in a minimal context. The reasons why graph rules can be in conflict are threefold:

1. One rule application deletes a graph item which is in the match of another rule application (delete-use conflict).

2. One rule application produces a graph item that gives rise to a graph structure that is forbidden by a NAC of another rule application (produce-forbid conflict).

3. One rule application changes attributes being in the match of another rule.

If all critical pairs can be resolved such that they can lead to the same result, the transformation system is called locally confluent. A locally confluent and terminating transformation system is confluent in general and thus shows a functional behavior [13].

Considering the critical pairs for our model transformation rules, we only have produce-forbid conflicts since all of our rules are non-deleting [17]. Let us have a look at Fig. 35, 


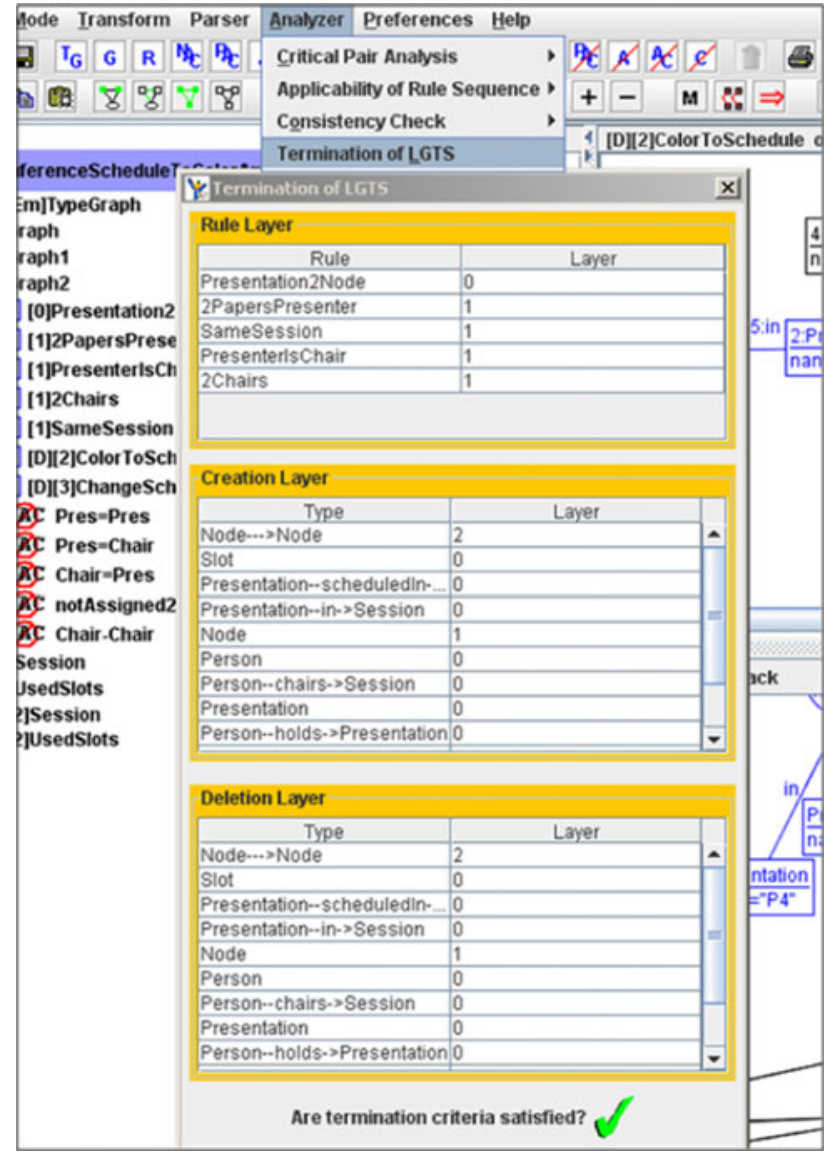

Fig. 34 Checking the model transformation for termination

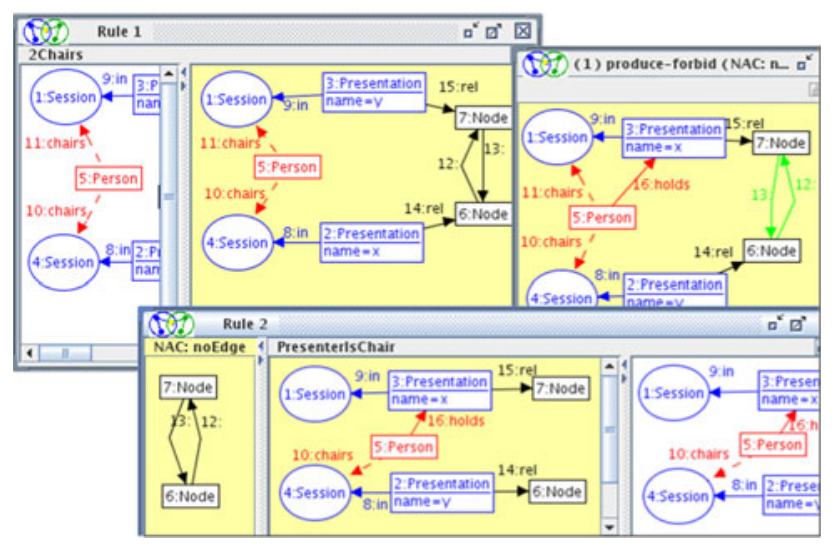

Fig. 35 A critical pair of rules 2Chairs and PresenterIsChair

showing a critical pair found for rules 2Chairs and PresenterIsChair.

The critical pair informs us that there may be the situation that a person may chair two sessions and hold a presentation in one of these sessions. In this situation, rule 2Chairs inserts a bidirectional edge (two edges, in fact) between Node 6 and Node 7 which prevents the application of rule PresenterIsChair afterwards. This is because the NAC of Presen-
terIsChair forbids the bidirectional edge. The critical part in the overlapping (the bidirectional edge between Node 6 and Node 7) is high-lighted in the conflict window to the upper right of Fig. 35. But analyzing the conflict, we find out that the effect of rule PresenterIsChair would have been the insertion of the bidirectional edge which has now been inserted by rule 2Chairs. So, preventing the application of rule PresenterIsChair after rule 2Chairs has been applied, means that we simply have more than one possibility to insert this bidirectional edge, and we can choose one of them non-deterministically and come to the same result. All of the remaining critical pairs of our graph transformation system describe analogous situations. Hence, we conclude that our graph transformation system is locally confluent, and having termination, it is also confluent.

Analogous observations can be made for the backward model transformation described in Sect. 4.3.4.

\section{Related work}

\subsection{AGG}

AGG is one of the standard graph transformation tools implementing the algebraic graph transformation approach as presented in [13]. Other graph transformation tools, such as Fujaba [7,26], ViaTra [2,33], VMTS [6,22,19], GrGen $[32,16,14]$, and Groove [15,24], implement other kinds of graph transformation approaches. In the following, we highlight essential differences in these approaches from the AGG point of view.

Most of the graph transformations tools rely on a different notion of graph than AGG. While AGG supports edges with identity, the other tools mentioned consider edges as relations between nodes only. The differences are subtle, but essential: While a relation between two nodes can be inserted at most once, an arbitrary number of edges with identity can be created between the same two nodes. If that is not desired, a corresponding constraint can be added. Thus, AGG is based on an elaborated graph notion which can be used in a flexible manner.

Next, the kinds of graph transformations which can be performed in the various tools also differ. AGG is characterized by a powerful transformation approach: matches as well as rules may be injective or non-injective. Non-injectivity means that two graph elements (nodes or edges) may be mapped to one graph element in the target graph. Thus, rules can express the gluing of graph elements and they can be flexibly applied to instance graphs. If nodes shall be deleted, potentially dangling edges either forbid a rule application or are also deleted. The rule designer can decide the intended semantics. Furthermore, any number of NACs may be added to a rule to optimally control its application context. Non-in- 
jectivity in NACs can express that two graph elements must not be mapped to one and the same element.

AGG hardly supports explicit control structure on rules. The reasons for this design decision are two-fold: On the one hand, the implementation of AGG goes hand-in-hand with the corresponding theory development and theory for controlled graph transformation is nearly not existing. On the other hand, AGG has an encapsulated graph transformation engine which can be used without the graphical user interface. It is possible and quite easy to write a program which reads in a graph e.g. from a GGX-file, computes a controlled graph transformation, and reports the result e.g. by writing it to a GGX-file again. This way any Java-control constructs can be used to control the application of rules.

VIATRA, Groove, and AGG are graph transformation tools which allow some kind of verification of graph transformation systems. While VIATRA and Groove concentrate on some kind of model checking, AGG is the only one which consequently implements the theoretical results available for algebraic graph transformation. These results are mainly concerned with conflict and dependency detection of rules based on critical pair analysis, termination checks, and recently also with static applicability checks for rule sequences [18].

\subsection{EMF Tiger}

EMF TIGER directly transforms EMF models [10]. Related model transformation approaches for EMF are mainly ATL (ATLAS Transformation Language [1]), Procedural and Declarative QVT (Operational and Relational [20]), and Xpand and Xtend (openArchitectureWare [23]).

EMF TIGER as well as all of the above mentioned frameworks support both: exogenous model translations between two different languages and endogenous transformations within the same language. Typical applications for the latter are model refactorings [3], reconfigurations, and operational semantics for modeling languages.

Compared to the existing model transformation approaches, endogenous transformations in particular can be very elegantly described with EMF Tiger and executed efficiently, since the transformations are applied in-place, i.e. directly to an existing instance model. No deep-copying is required (as opposed to ATL). Moreover, EMF TIGER offers an intuitive graphical notation and uses a light-weight underlying transformation model. In contrast to endogenous transformations, exogenous (source-to-target) model translations require an additional reference model that keeps track of newly created elements and their relation to original ones. Note that the transformation engine itself is oblivious to the type of transformation performed, i.e. exogenous and endogenous transformations are treated equally by the transformation engine.
The transformation language of EMF Tiger has a declarative nature. The underlying transformation engine relies on graph-based pattern matching. Modifications of instance models are always performed in-place. Furthermore, EMF TIGER provides a converter which translates EMF transformation rules as well as ECORE and instance models to AGG.

As shown in [5], a slightly restricted form of EMF model transformations can be formalized as attributed graph transformations and thus, can be subject to verification by AGG. Together with the native graphical notation, this further distinguishes EMF TIGER from related existing approaches.

\section{Evaluation and conclusion}

The Conference Scheduling case study offers quite a diversity of modeling and analysis challenges.

Introducing our two tools AGG and EMF TIGER, we aimed to give concrete evidence that the modeling and analysis techniques we support, matter in practice. In particular, we concentrated on the case aspects, where our approaches are most useful, which are the flexibility in modeling, analysis and visualization. The following list gives a summary of the main aspects of the case study and points out how far our tools managed to deal with them:

- Meta-modeling. Both AGG and EMF TIGER use metamodeling to describe the problem domain: AGG uses type graphs with inheritance and EMF TIGER uses EMF models (.ecore format). In both cases, attribution is supported. The meta-models guarantee syntactic correctness of the instance model at any step of the transformation (Sect. 4.1.1).

- Usability of the editors. Both tools offer visual editors (abstract syntax based) for rule graphs, NACs and mappings. Mappings between graph objects are visualized by node colors and/or by numbering. In AGG, graph constraints are used to check system behaviour properties (invariants). Graph constraints are used both at rule level and at system level, e.g. to ensure that the computed result is valid.

- Matching. Partially automatic matching is supported both in AGG and EMF TIGER, where a (part of the) match can be defined visually in AGG, and can be set manually in the generated Java code that applies the rule in EMF TIGER. Non-deterministic and non-injective matching is possible in both tools. For fully automatic simulation runs, predefined matches can be defined in EMF TIGER using the generated Java methods for match definition (Sect. 4.1.4).

- Rule application control. Rule application control is offered by both tools, where AGG mainly uses NACs, attribute conditions and layers for rule structuring. (As 
indicated in Sect. 5.1, AGG also offers the possibility to define rule control by Java code). Using the graphical user interface of AGG, the modeler is allowed to define loops over layers depending on the satisfaction of previously defined graph constraints (Sect. 4.1.5). EMF TIGER controls rule application more flexibly by Java code which uses the generated rule application code. Hence, rule control is not limited by the tool, a fact which has been heavily exploited in this case study modeling the assignment instance generation (Sect. 4.4).

- Visualization of transformations. AGG shows simulation runs in the visual editor panel of the host graph, where not only the start graph can be drawn but also rule application effects are shown at runtime (based on the abstract visual syntax). Various graph layouting options are offered for tuning the visualization to become as appealing and understandable as possible (Sect. 4.1.3). A drawback of EMF TIGER right now is a missing visual debugger consisting of a visual viewer for instance graphs and the possibility to define breakpoints and perform stepwise transformations. In the current state, transformation results are EMF instance models which can be shown e.g. in XML format (see Fig. 14). In many cases, an adequate visualization solution would be the possibility to define a concrete syntax which can be used not only to show the resulting system graph but also to define the rewrite rules, as it is realized in our TIGER editor designer and generator [30], a tool based on graph transformation and the graphical editing framework GEF of ECLIPSE.

- Analysis. Here is the strong point of AGG, which supports formal analysis like conflict and dependency detection of rules based on critical pair analysis, and termination checks (Sect. 4.4). The adaption of comparable analysis techniques to EMF transformation in EMF TIGER is work in progress.

- Interoperability. AGG has been extended by the possibility to export a graph into the DIMACS format of coloring algorithms, and to import the result, given in the same format (Sect. 4.3). This extension of AGG is the only solution part which was not realized during the live contest but later. AGG supports GXL, an exchange format supported by several graph-based tools. Hence, a more general interoperability approach (not assuming knowledge of AGG internals) would be to define an XSLT stylesheet [34] which transforms any GXL graph to DIMACS and back. Since EMF TIGER uses the EMF (Ecore) model format, interoperability is supported by the ECLIPSE plug-in technology; especially for EMF modelling, plug-ins for various modelling purposes exist (see EMF Technology at [11]).

- Performance. Previous tool contests have shown that one field for improvement is the performance. Here, graph transformation tools like GrGen or VIATRA 2 have proven to be able to compute solutions in much shorter time (see [28] for a benchmark of graph transformation tools for the Sierpinsky triangle case study). We determined the performance for our tools for the conference scheduling case study by computing the runtime of 500 runs searching solutions for the given scheduling example (see Fig. 15). EMF TIGER needed 3789 ms (7.6 ms for one average run), and AGG needed $14103 \mathrm{~ms}$ for 500 runs (28.2 ms for one average run). These numbers are to give a rough orientation of the performance of our tools. They were computed using standard hardware (a notebook with 2 Gigahertz (dual core) and 2 GB memory). We are aware that the bottleneck in our tools is the match-finding process. We plan to use search plans and heuristics in future to achieve accelerations here.

- Scheduling algorithm. We used a set-and-test procedure to compute scheduling solutions, i.e. we start the computation procedure again when we do not find a valid solution due to unlucky session assignments earlier in the scheduling procedure. Here, backtracking would help not to start at the very beginning each time, but to try systematically other schedules. AGG has a backtracking mechanism already built for parsing of visual languages. We plan to make this algorithm available also for general graph transformation systems. In EMF Tiger, backtracking should be provided at rule control level.

Although the features of both tools seem quite similar at first sight (visual transformation rule modeling, metamodeling, non-deterministic rule application, or application controlled by well-defined control structures), the main difference of the key concepts (EMF model manipulation for EMF TIGER and typed attributed graph transformation techniques for AGG) lead to the following recommendation when to use which tool: the strength of EMF TIGER is its ability to work with existing EMF meta-models whereas AGG is strong in the static analysis of properties of graph transformation systems (e.g. conflicts, dependencies, applicability of rule sequences).

Acknowledgments The authors would like to thank the organizers of the GraBaTs'08 tool contest, Arend Rensink and Pieter Van Gorp for their efforts. We also want to thank the anonymous referees for their valuable hints on this paper.

\section{References}

1. ATL: The Atlas Transformation Language Home Page. http://www. sciences.univ-nantes.fr/lina/atl (2006)

2. Balogh, A., Németh, A., Schmidt, A., Rath, I., Vágó, D., Varró, D., Pataricza, A.: The VIATRA2 model transformation framework. In: Proceedings of the European Conference on Model Driven Architecture (ECMDA'05) (2005) 
3. Biermann, E., Ehrig, K., Köhler, C., Kuhns, G., Taentzer, G., Weiss, E.: EMF Model Refactoring based on Graph Transformation Concepts. In: Proceedings of the Software Evolution through Transformations (SETra'06), vol. 3, Electronic Communications of the EASST (2006)

4. Biermann, E., Ehrig, K., Köhler, C., Kuhns, G., Taentzer, G., Weiss, E. : Graphical definition of in-place transformations in the eclipse modeling framework. In: Nierstrasz, O., Whittle, J., Harel, D., Reggio, G. (eds.) Proceeding of the International Conference on Model Driven Engineering Languages and Systems (MoDELS'06). LNCS, vol. 4199, pp. 425-439. Springer, Berlin (2006)

5. Biermann, E., Ermel, C., Taentzer, G.: Precise semantics of EMF model transformations by graph transformation. In: Czarnecki, K. (ed.) Proceedings of the International Conference on Model Driven Engineering Languages and Systems (MoDELS'08). LNCS, vol. 5301, pp. 53-67. Springer, Berlin (2008)

6. Budapest University of Technology and Economics, HUN. Visual Modeling and Transformation System (VMTS) (2008). http:// www.aut.bme.hu/Portal/Vmts.aspx?id=da39f049-9963-40d7ba92-fdac9e0e479d

7. Burmester, S., Giese, H., Hirsch, M., Schilling, D., Tichy, M.: The Fujaba real-time tool suite: model-driven development of safetycritical, real-time systems. In: Proceedings of the International Conference on Software Engineering (ICSE'05), pp. 670-671. ACM Press, New York (2005)

8. Culberson, J.C.: Iterated greedy graph coloring and the difficulty landscape. Technical Report TR 92-07, Department of Computing Science, University of Alberta, Edmonton, Alberta, Canada (1992)

9. Culberson, J.C.: Graph Coloring Programs Manual. (2009). http://www.cs.ualberta.ca/ joe/Coloring/Colorsrc/manual.html

10. Eclipse Consortium (2008) Eclipse Modeling Framework (EMF)_Version 2.4. http://www.eclipse.org/emf

11. Eclipse Consortium (2009) Eclipse Modeling Framework Technology. http://www.eclipse.org/modeling/emft

12. Ehrig, H., Ehrig, K., de Lara, J., Taentzer, G., Varró, D., VarróGyapay, S.: Termination criteria for model transformation. In: Wermelinger, M., Margaria-Steffen, T. (eds.) Proceedings of the Fundamental Approaches to Software Engineering (FASE'05). LNCS, vol. 2984, pp. 214-228. Springer, Berlin (2005)

13. Ehrig, H., Ehrig, K., Prange, U., Taentzer, G.: Fundamentals of Algebraic Graph Transformation. EATCS Monographs in Theor. Comp. Science. Springer, Berlin (2006)

14. Geï̈, R., Batz, G., Grund, D., Hack, S., Szalkowski, A.M.: Grgen: a fast SPO-based graph rewriting tool. In: Corradini, A., Ehrig, H., Montanari, U., Ribeiro, L., Rozenberg, G. (eds.) Proceedings of the International Conference on. Graph Transformation (ICGT'06). LNCS, vol. 4178, pp. 383-397. Springer, Berlin (2006)

15. GRaphs for Object-Oriented VErification (GROOVE) (2008) http://groove.sourceforge.net/groove-index.html

16. Jakumeit, E., Buchwald, S., Kroll, M.: Manual and automated performance optimization of model transformation systems. Int. J. Softw. Tools Technol. Transfer (2010). doi:10.1007/s10009-0100148-8

17. Lambers, L., Ehrig, H., Orejas, F.: Conflict detection for graph transformation with negative application conditions. In: Proceedings of the International Conference on Graph Transformation (ICGT'06). LNCS, vol. 4178, pp. 61-76. Springer, Berlin (2006)

18. Lambers, L., Ehrig, H., Taentzer, G.: Sufficient criteria for applicability and non-applicability of rule sequences. In: de Lara, J., Ermel, C., Heckel, R. (eds.) Proceedings of the Workshop on Graph
Transformation and Visual Modeling Techniques (GT-VMT'08). Electronic Communications of the EASST, vol. 10 (2008)

19. Levendovszky, T., Lengyel, L., Mezei, G., Charaf, H.: A systematic approach to metamodeling environments and model transformation systems in VMTS. In: Proceedings of the Workshop on Graph-Based Tools (GraBaTs'04). ENTCS, vol. 127(1), pp. 6575. Elsevier Science, Amsterdam (2005)

20. Model To Model: The M2M subproject of the Eclipse Modeling Project, (2009) http://www.eclipse.org/m2m/

21. Mens, T., Van Gorp, P., Varrò, D., and Karsai, G.: Applying a model transformation taxonomy to graph transformation technology. In: Proceedings of the Workshop on Graph and Model Transformation (GraMoT'05). ENTCS, vol. 152, pp. 143-159. Elsevier Science, Amsterdam (2005)

22. Mészáros, T., Mezei, G., Levendovszky, T., Asztalos, M.: Manual and automated performance optimization of model transformation systems. Int. J. Softw. Tools Technol. Transfer (2010). doi:10.1007/ s10009-010-0151-0

23. OpenArchitectureWare: the leading platform for professional model-driven software development (2009) http://www. openarchitectureware.org/

24. Rensink, A.: The GROOVE simulator: a tool for state space generation. In: Pfalz, J., Nagl, M., Böhlen, B. (eds.) Applications of Graph Transformations with Industrial Relevance (AGTIVE). LNCS, vol. 3062, pp. 479-485. Springer, Berlin (2004)

25. Rensink, A., Van Gorp, P.: Graph transformation tool contest 2008. Int. J. Softw. Tools Technol. Transfer (2010). doi:10.1007/s10009010-0157-7

26. Software Engineering Group, University of Paderborn. Fujaba Tool Suite (2007). http://wwwcs.uni-paderborn.de/cs/ag-schaefer/ Lehre/PG/Fujaba/projects/tgg/index.html.

27. Taentzer, G.: AGG: a graph transformation environment for modeling and validation of software. In: Pfaltz, J., Nagl, M., Boehlen, B. (eds.) Application of Graph Transformations with Industrial Relevance (AGTIVE'03). LNCS, vol. 3062, pp. 446-456. Springer, Berlin (2004)

28. Taentzer, G., Biermann, E., Bisztray, D., Bohnet, B., Boneva, I., Boronat, A., Geiger, L., Geï̈, R., Horvath, A., Kniemeyer, O., Mens, T., Ness, B., Plump, D., Vajk, T.: Generation of sierpinski triangles: a case study for graph transformation tools. In: Schürr, A., Nagl, M., Zündorf, A. (eds) Proceedings of International Symposium on Applications of Graph Transformation with Industrial Relevance (AGTIVE'07). LNCS, vol. 5088, pp. 514-539. Springer, Berlin (2008)

29. TFS-Group, Technische Universität Berlin. AGG (2009). http://tfs. cs.tu-berlin.de/agg

30. TFS-Group, Technische Universität Berlin. Tiger: Generating Visual Environments in Eclipse (2005). http://tfs.cs.tu-berlin.de/ tigerprj

31. TFS Group, Technische Universität Berlin. EMF Tiger (2009). http://tfs.cs.tu-berlin.de/emftrans

32. Universität Karlsruhe. Graph Rewrite GENerator (GrGen) (2006). http://www.info.uni-karlsruhe.de/software.php/id=7\&lang=en

33. VIATRA2 (VIsual Automated model TRAnsformations) framework (2006). http://dev.eclipse.org/viewcvs/indextech. cgi/ checkout $/$ gmt-home/subprojects/VIATRA2/index.html

34. WWW Consortium (W3C) XSL Transformations (XSLT) Version 2.0 (2007). http://www.w3.org/TR/xslt20/ 